\title{
VIRTUAL REALITY USER INTERFACE FOR AUTONOMOUS PRODUCTION
}

\author{
Christopher Schlick, Ralph Reuth, Holger Luczak \\ Institute of Industrial Engineering and Ergonomics \\ Aachen University of Technology \\ c.schlick@iaw.rwth-aachen.de
}

\begin{abstract}
This paper deals with design, implementation, and evaluation of a virtual reality user interface for autonomous production systems. Tthe conceptual design of the application layer, the dialog layer, and the physical interaction layer is presented and a user interface prototype for autonomous 3D-laser welding, so called ActiveUI, is introduced. The usability of ActiveUI is tested with the help of an empirical study with nine laser welding experts concerning (1) suitability for the task, (2) self-descriptiveness, (3) controllability and (4) conformity with user expectations. Based on this study design recommendations for future user interfaces are identified and discussed.
\end{abstract}

\section{INTRODUCTION}

In order to succeed with high-quality products in turbulent and highly networked world markets the European industry has a great demand for highly adaptive manufacturing systems. Cybernetic background of this thesis is Conant \& Ashby's (1970) law of requisite variety, which states that every good regulator of a system must be a model of that system. Hence, future manufacturing systems should focus on the integration of the human operator as the most adaptive ,regulator" due to his extraordinary problem solving abilities, as well as learning and communication skills. Following this paradigm, the approach of Autonomous Production Cells (APC) was developed at Aachen University of Technology with a strong focus on operator empowerment. The three core design criteria of APC are defined as: (1) Human operator and technical subsystems develop a cellular organizational unit within the organism of flexible production plants; (2) Planning, set-up, NCprogramming, process control, quality inspection and fault management tasks are integrated locally on the shop floor; (3) Operator's task spectrum is fully supported by means of user-centered design of decision support and information systems.

\section{CONCEPTUAL DESIGN}

The following sections focus on the user interface design of APC's numerical control for 3D-laser welding, because this is an innovative material processing 
technology which also has a great market potential. The prototype of the user interface is called ActiveUI and was developed in cooperation with the Institute of Lasertechnology. ActiveUI relies on virtual reality displays as well as speechprocessing. To structure the design process a three-layer interface architecture was chosen, which distinguishes the application layer, the dialog layer, and the physical interaction layer.

\subsection{Application Layer}

For the design of ActiveUI the metaphoric principle was preferred and the metaphor of a virtual autonomous welding cell was created. With the help of this metaphor an informational structure is developed, which empowers the APC operator to execute his tasks effectively and efficiently. According to Hutchins (1989) this approach represents a "model world" with a focus on the physical form and physical function of the production machine on the shop floor. With regard to semantics of metaphoric user interface objects the class diagram of the Unified Modeling Language (UML, Booch et al., 1998) is shown in figure 1. According to figure 1 the seven userconfigurable sensors embedded in ActiveUI are: (1) raw beam monitor (RBM) to control the power density function of the raw laser beam; (2) focus beam monitor (FBM); (3) plasma shielding control (PSC), which shields the quality-critical plasma propagation; (4) fast laser power monitor (FLPM); (5) wire feed (WF); (6) temperature control (TC); (7) through-welding control (TWC). The following five tasks of the APC operator are supported by the metaphor: (1) Setup of robot and sensors; (2) selection of manufacturing order; (3) workshop-oriented simulation; (4) process control and monitoring; (5) process diagnosis.

\subsection{Dialog Layer}

Following Shneiderman (1998), the principle of direct manipulation was preferred for the dialog design of ActiveUI. Thus, the user can rely on rule-based behavior (Rasmussen, 1986) for the discrete-state control of interface objects and use his skill-based behavior for spatial and temporal interaction with the model world. Moreover, electronic forms were used to specify sensor parameters and button panels served as interface controls for task sequencing as well as dynamic dialog behavior. ActiveUI has two virtual reality displays, which can be controlled via direct manipulation: First, the Macro-display, which is an exact representation of the welding robot and uses symbols of sensors and the workpiece (figure 2). This display is used for robot and sensor setup as well as to select the manufacturing order. Second, the Micro-display, which represents the shape of the workpiece exactly and is useful for workshop-oriented simulation, process monitoring and diagnosis. In the Micro-display the user can directly point to segments of the virtual weld seam and the corresponding sensor signals are visualized with the help of an electronic line chart of sensor data (figure 3).

\subsection{Physical Interaction Layer}

ActiveUI's physical interaction design is based on two-hand manipulation of the Macro- and Micro-displays. In order to simplify hand-eye coordination the preferred 
hand uses a lightpen to manipulate the states of user interface object directly on screen. The other hand controls the viewpoint in terms of an isotonic first-order system with a six-degrees-of-freedom mouse. Movements in the $x$-y-plane were blocked by software, so that the spatial interaction in real-time is easier for novices. Alphanumerical symbols can be input via a discrete speech-processing unit, which is speaker independent. Thus, ActiveUI can be used without a keyboard.

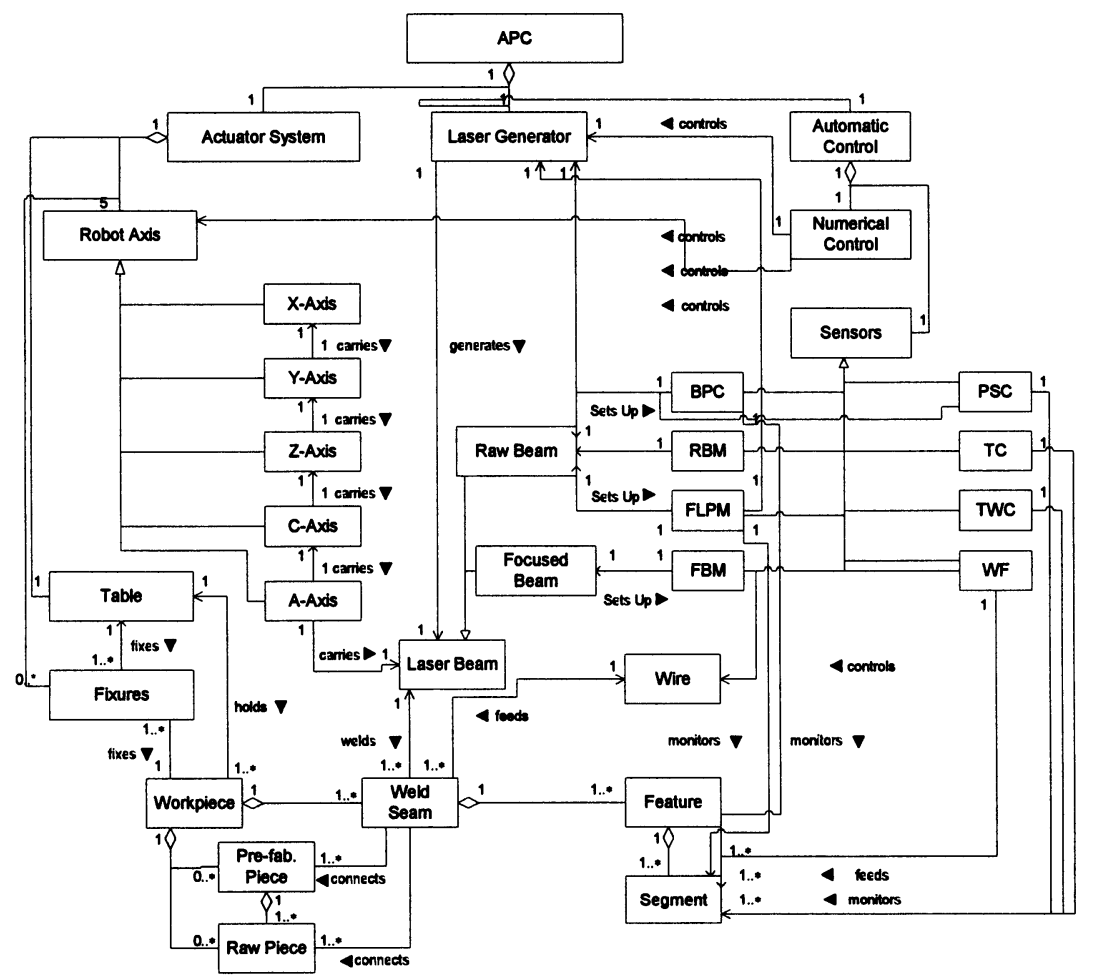

Figure 1 - Class diagram according to metaphor of a virtual laser welding cell

\section{IMPLEMENTATION}

For the implementation of ActiveUI two software processes were coded. The first process renders the virtual world and integrates the physical interaction devices. The second process controls the states of the user interface and communicates with the robot control of the production cell. The inter-process communication relies on TCP/IP and uses a self-developed protocol. The first process was realized with the help of virtual reality libraries from Sense8 and is written in C++. The second process is also written in $\mathrm{C}++$ and uses the Microsoft foundation classes. Moreover, Dragon Systems development kit delivered the speech-engine. An important implementation goal was preemptive usability. That means, dialog threads can occur simultaneously and only functional dependencies of the user interface are blocked. 


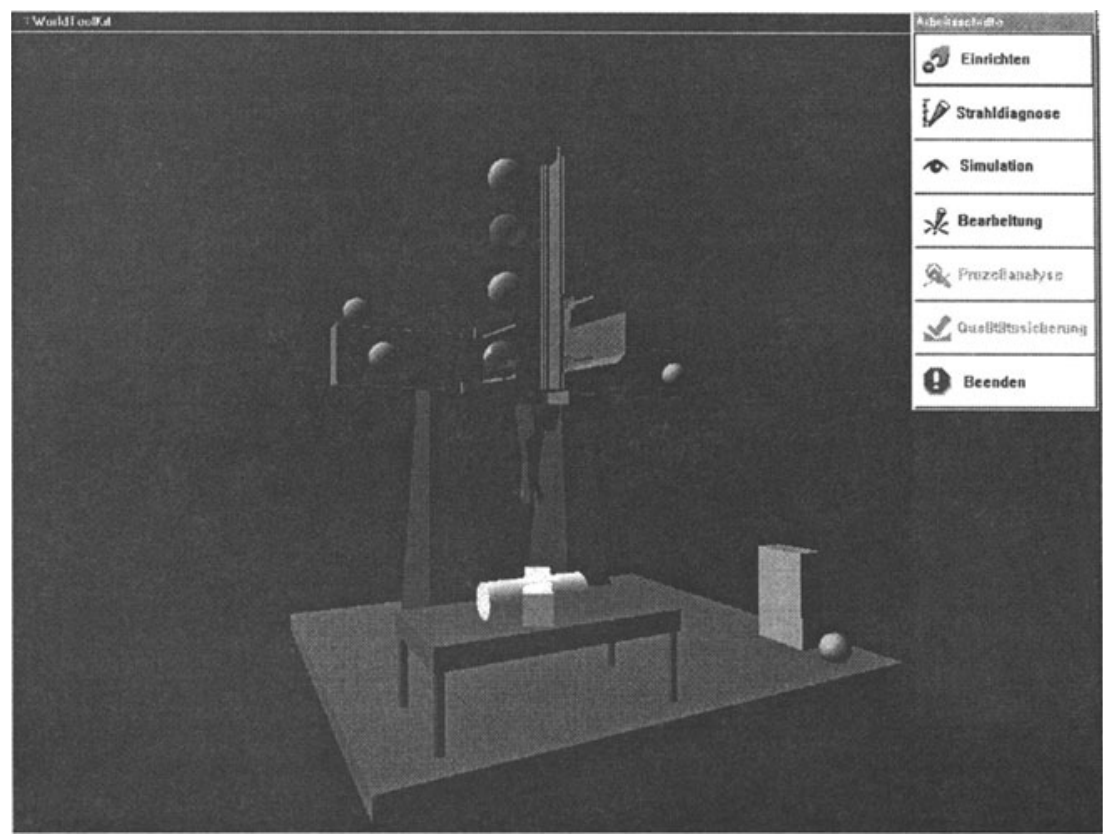

Figure 2 - Screenshot of ActiveUI's Macro-display for robot setup

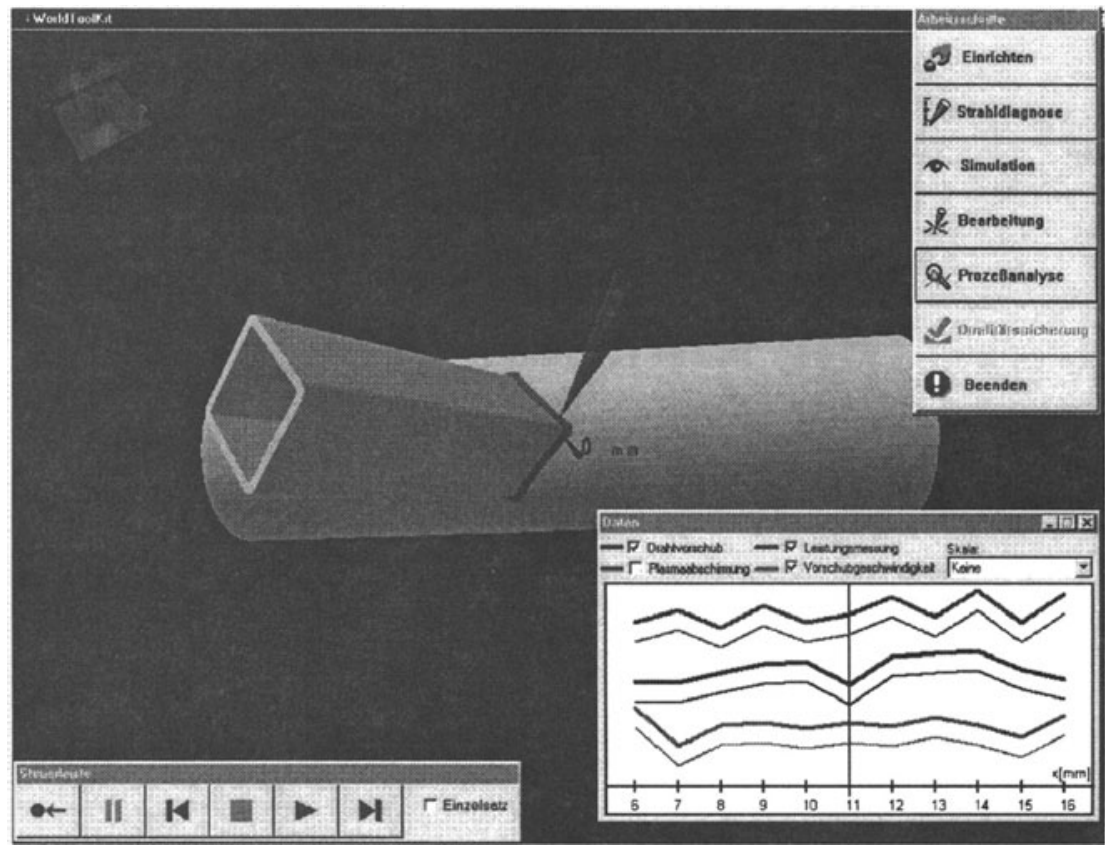

Figure 3 - Screenshot of Micro-display for process simulation and diagnosis 


\section{USABILITY TESTING}

\subsection{Methods and Procedures}

Four usability criteria of the ISO 9241 (Part 10) standard were investigated empirically: (1) suitability for the task, (2) self-descriptiveness, (3) controllability and (4) conformity with user expectations. Therefore a laboratory study was carried out. The experimenter gave the user standardized interaction tasks and the interaction behavior was observed and recorded on videotape. In addition, the input activities like the trajectory in the model world as well as state transitions of user interface objects were written into a logfile. The experimental layout is sketched in figure 4. A total of nine experts in 3D-laser welding participated in a 100-minute study. Their age varied from 26 to 44 years $(\bar{x}=32,7 \mathrm{a} ; \mathrm{s}=6,0$ a) and the professional experience varied from 1 to 6 years $(\bar{x}=3,6 \mathrm{a} ; \mathrm{s}=1,9 \mathrm{a})$.

The usability testing was carried out in five steps. First, the self-descriptiveness of ActiveUI was tested. Therefore, the user had to explain certain objects of the virtual welding cell. Dependent variable was the relative number of correctly recognized interface objects. Second, the conformity with user expectations was investigated for subtasks like sensor setup and loading the NC-program. Moreover, the mental representation of the button panel for the selection of working steps (cf. figure 2) was acquired. Dependent variable was the relative number of correctly expected functions. Third, the controllability was tested. Therefore user's actions for certain subtasks, e.g. start of workshop-oriented simulation and process analysis, were observed. For each replication the level of correctness was rated by the experimenter. Dependent variable was the relative number of correctly controlled subtasks. Fourth, the suitability for the task was focused and the user's experimental task was to execute a complete welding order with ActiveUI. The working steps were observed, cognitive bottlenecks as well as human errors were documented and a standardized questionnaire was carried out. Fifth, the semantic self-descriptiveness was investigated with the help of a participatory structure modeling technique. Therefore the user had to layout a entity-relationship diagram of his mental representation of the virtual welding cell. Reference structure was the class diagram of figure 1. Dependent variable was a "semantic quotient" $q$, which assessed the relationships among the reference structure and user's diagram ( $q=1$ - ((number of false relationships + number of missing relationships) $/$ total number of relationships)) ).

\subsection{Results and Discussion}

Concerning the first evaluation step the mean number of correctly recognized and therefore self-descriptive interface objects of the Macro-display was $46 \%(\mathrm{~s}=6 \%)$. The detailed distribution is shown in figure 5, first bar. This quite low recognition rate is due to insufficient symbols of the sensors. If theses symbols are excluded, the mean number of self-descriptive objects is increased to satisfactory $79 \%$ (figure 5 , second bar). Hence, the first design recommendation is to improve the symbols by functional-related shaping. 
In the second step the mean number of correctly answered attributes of sensor functions was $78 \%$ (figure 5 , third bar) with a standard deviation of $11 \%$. Hence, although the static self-descriptiveness of sensors was only $46 \%$ the dependent variable increases to $78 \%$ for real-time interaction. That means, ActiveUI's dynamics need not to be changed. Nevertheless, the deactivation of sensors was correctly anticipated by three users only and therefore the second design recommendation is to simplify the sensor deactivation sequence. The button panels for the selection of working steps had a very good user response, because only 4 from a total of 63 answers were incorrect.

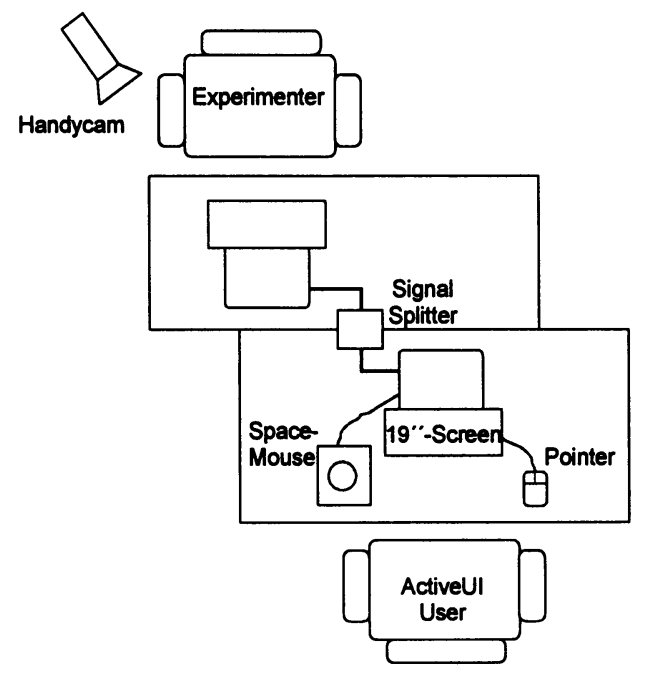

Figure 4 - Sketched arrangement in the evaluation.

In the third step the investigation of the ActiveUI's controllability showed, that a mean of $93 \%(s=8 \%)$ of the subtasks were finished correctly by the users. This high success rate proves a good controllability of both displays - Macro-display as well as Micro-display. The distribution of the dependent variable is shown in figure 5 , fourth bar. In spite of this positive result, the third design recommendation is to use the electronic line chart for sensor data directly for the selection of segments on the virtual weld seam, in addition to the direct manipulation of the virtual workpiece in the Micro-display. Therefore scroll bars or a panning function should be integrated.

With regard to the fourth step all nine users were able to process a welding order - from sensor setup to quality control - completely and correctly with ActiveUI. However, some usability problems arouse concerning the incremental activation of single sensors. Hence, the fifth design recommendation is to integrate a "grouping" function for manipulation of a sensor set as a whole. The results of the standardized questionnaire are summarized in table 1.

Finally, the test of transparency demonstrated a mean semantic quotient $\bar{q}$ of $0,46(\mathrm{~s}=0,08)$. The distribution is shown in figure 5 , fifth bar. That means, ActiveUI's metaphoric approach is sufficiently able to develop the mental model of 
the user during the average 100 minutes of usability testing. Here, representation problems arouse concerning the virtual weld seam and functional dependencies among sensors. Hence, the sixth design recommendation refers to a multi-layered application architecture: The virtual world should be modeled on hierarchical semantic layers - e.g. physical form, physical functions up to order processing functions - in order to increase the mental compatibility.

Table 1 - Results of questionnaire of ActiveUI's task suitability ( $\mathrm{n}=9$ laser experts)

\begin{tabular}{|l|l|}
\hline Aspect & Assessment \\
\hline 1. During laser welding I am able to control the sensor parameters & Good \\
\hline 2. I am able to assess the welding process quality online & Sufficient \\
\hline 3. I am able to decide when to intervene the laser welding process & Satisfactory \\
\hline 4. I can setup the sensors, so that relevant parameters are represented & Good \\
\hline 5. I can intervene the working steps directly & Sufficient \\
\hline 6. I am able to asses the weld seam quality offline & Satisfactory \\
\hline
\end{tabular}

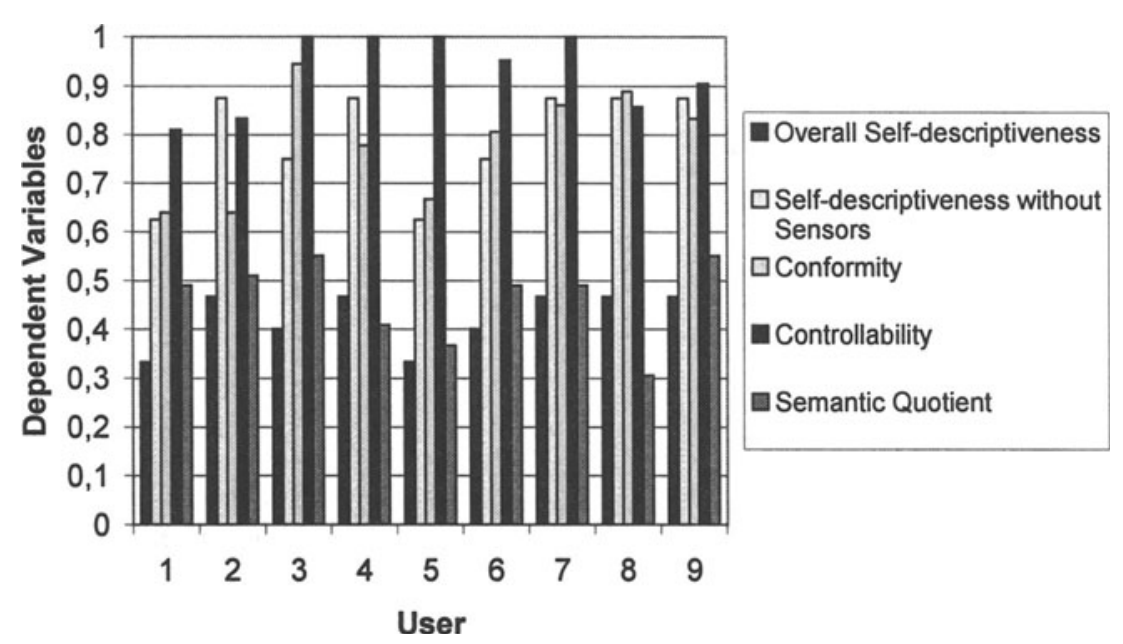

Figure 5 - Summarized results of usability testing $(n=9$ laser experts)

\section{CONCLUSION AND OUTLOOK}

In summary, the cited design recommendations can be covered by two major user interface revisions: First, ActiveUI should integrate a redundant option for sensor 
setup besides the direct-manipulative mode of the Macro-display. This option should refer to an additional setup button panel, should improve the static transparency of sensor symbols and should integrate a function for grouping of sensors. Moreover, the functional dependencies among subsystems of the welding cell should be represented on different abstraction layers in terms of an means-ends abstraction hierarchy (Rasmussen, 1986). Second, the electronic line graph of sensor parameters should be fully integrated into ActiveUI's Micro-display. That means, the planned and the measured sensor data should be visualized directly in conjunction with the virtual weld seam. Therefore a compensatory display mode is appropriate and moreover, due to the multi-dimensionality of seven sensor signals the approach of polygon-displays (Greany \& MacRae, 1996) seems promising.

Finally, the usability testing demonstrated a very high robustness of the software prototype. Because of the systematic design and implementation, ActiveUI neither crashed nor generated protection violations during the usability tests in spite of its rich interaction functionalities. This aspect is not covered by the ISO 9241 standard, but is an important aspect for the user concerning an overall assessment of interface quality.

In the long run, it is planned to integrate additional user interface innovations such as augmented reality displays (Azuma, 1997) or interface agents (Bradshaw, 1997). With regard to interface agents a first step already carried out with the help of Bayesian Networks to stochastically represent user's goals and intentions (Kuenzer, 1999). However, from the point of view of human factors such mixed-initiative interfaces are a "quantum leap" from contemporary design paradigms and therefore a lot of basic research has to be done to ensure a high level of efficiency, effectiveness and satisfaction.

\section{ACKNOWLEDGEMENTS}

The research is funded by the German Research Foundation according to the collaborative research center no. 368 "Autonomous Production Cells".

\section{REFERENCES}

Azuma, R.T.: A survey of augmented reality. Presence 6 (1997), 4, pp. 355-385.

Booch, G.; Rumbaugh, J.; Jacobson, I.: The Unified Modeling Language User Guide. Reading (MA): Addison Wesley 1998.

Bradshaw, J.M. (Ed.): Software Agents. Cambridge (MA): MIT Press 1997.

Conant, R. G.; Ashby, W. R.: Every good regulator of a system must be a model of that system. International Journal of Systems Science 1 (1970), 2, pp. 89-97.

Greany, J.; MacRae, A.N.: Diagnosis of Fault Location Using Polygon Display. Ergonomics 39 (1996), 3, S. $400-411$.

Hutchins, E.: Metaphors for Interface Design. In: Taylor, M.; Neel, F.; Bouwhuis, D. (Eds.): The Structure of Multimodal Dialogue. Amsterdam: Elsevier Science 1989.

Kuenzer, A.: Gestaltung eines Interface-Agenten fur eine Benutzungsschnittstelle der virtuellen Realităt. Master Thesis, Aachen University of Technology 1999.

Rasmussen, J.: Information Processing and Human-Machine Interaction. An Approach to Cognitive Engineering. New York (NY): North-Holland 1986.

Shneiderman, B.: Designing the User Interface. Third edition. Reading (MA): Addison Wesley 1998. 